\title{
Feedback Systems using Non-Binary LDPC Codes with a Limited Number of Transmissions
}

\author{
Kasra Vakilinia, Adam R. Williamson, Sudarsan V. S. Ranganathan, Dariush Divsalar*, and Richard D. Wesel \\ Department of Electrical Engineering, University of California, Los Angeles, Los Angeles, California 90095 \\ *Jet Propulsion Laboratory, California Institute of Technology, Pasadena, California 91109
}

\begin{abstract}
One advantage of incremental transmissions with feedback in point-to-point memoryless channels is a reduction in average blocklength required to approach capacity. This paper optimizes the size of each incremental transmission for nonbinary (NB) LDPC codes to maximize throughput in VLFT and two-phase VLF settings. The optimization problem uses an approximation based on the inverse-Gaussian p.d.f. of the blocklength required for successful decoding. By using the optimized incremental transmission lengths (with an average blocklength of less than 500 bits), NB-LDPC codes for VLFT setting limited to 5 transmissions achieve a throughput greater than $96 \%$ of that obtained by an unlimited-transmission VLFT scheme with the same average blocklength. With a similar average blocklength, a two-phase VLF system limited to five transmissions (with optimized lengths) using the binary image of NB-LDPC codes achieves greater than $90 \%$ of the capacity of binary-input AWGN channel with SNR=2 dB. Two-phase VLF does not match the throughput of VLFT, but it is more practical than VLFT because it does not assume noiseless transmitter confirmation.
\end{abstract}

\section{INTRODUCTION}

The classical results from [1] show that feedback does not increase the asymptotic capacity of memoryless channels. Polyanskiy et al. [2] and Chen et al. [3] illustrate that by using feedback, one can approach capacity in a small number of channel uses (i.e. with low latency). Polyanskiy et al. [2] introduce variable-length feedback coding with termination (VLFT) and without termination (VLF) which theoretically approach capacity with average blocklengths on the order of a few hundred bits. Without feedback, similar performance requires a capacity-approaching coding technique such as LDPC with blocklengths of several thousand bits.

In VLFT, the receiver provides full noiseless feedback to the transmitter. The transmitter sends additional incremental information over the channel until it determines that the receiver has correctly decoded. Termination, the "T" in VLFT, occurs when the transmitter sends a noiseless transmitter confirmation (NTC) to terminate the transmission. The NTC is sent through a noiseless channel separate from the primary communication channel. The transmitter's knowledge of the decoder state through feedback and the NTC together facilitate zero probability of error in VLFT.

This material is based upon work supported by the National Science Foundation under Grant Numbers 1162501 and 1161822. Any opinions, findings, and conclusions or recommendations expressed in this material are those of the author(s) and do not necessarily reflect the views of the National Science Foundation. This research was carried out in part at the Jet Propulsion Laboratory, California Institute of Technology, under a contract with NASA JPL Task Plan 82-17473.
In contrast to VLFT, in the VLF framework, the receiver determines when to stop the transmission and informs the transmitter via noiseless feedback. The stopping criterion that the receiver uses can be a CRC, a reliability metric about the decoded codeword as in [4], or a confirmation message from the transmitter through the primary communication channel as in [5]. This last scheme, with each communication phase followed by a confirmation phase, is called "two-phase" VLF.

Vakilinia et al. [6] show throughput versus latency for various non-binary (NB) LDPC codes in VLFT with unlimited transmissions. Additional incremental bits are selected one at a time, and decoding is attempted after each received bit.

This paper considers a more practical scenario in which the number of transmissions is limited. The size of the incremental messages in each transmission significantly affects the overall throughput of the system. We describe methods to select the size of each incremental message to maximize the throughput in VLFT and two-phase VLF schemes.

We approximate the probability density of the blocklengths that support successful decoding and the corresponding instantaneous rates via "inverse-Gaussian" and Gaussian probability density functions (p.d.f.) respectively. These approximations enable us to formulate an analytical throughput maximization problem that facilitates optimization of the sizes of the incremental transmissions.

We compare the throughput achieved by our optimization applied to NB-LDPC codes with convolutional codes whose blocklengths were optimized by a coordinate-descent algorithm in [7] for information blocks of $k=12,24$, and 36 $G F(256)$ symbols ( $k=96,192$, and 288 bits) with $m=5$ transmissions in VLFT and two-phase VLF settings.

The paper proceeds as follows: Sec. II provides an overview of the VLFT system with NB-LDPC codes and the inverseGaussian approximation for the histogram of the cumulative blocklengths. Sec. III presents the optimization techniques to select the size of each incremental transmission in VLFT. Sec. IV gives an overview of the two-phase VLF scheme and optimizes the cumulative blocklengths in each decoding attempt. Sec.V compares the throughput and the expected latency of NB-LDPC and convolutional codes in VLFT and two-phase VLF settings. Sec. VI concludes the paper.

\section{VLFT WITH NON-BINARY LDPC CODES}

In [6], Vakilinia et al. use NB-LDPC codes in a VLFT system with 1-bit increments. After the initial transmission, the 
p.d.f of blocklength until the decoder converges to the correct codeword

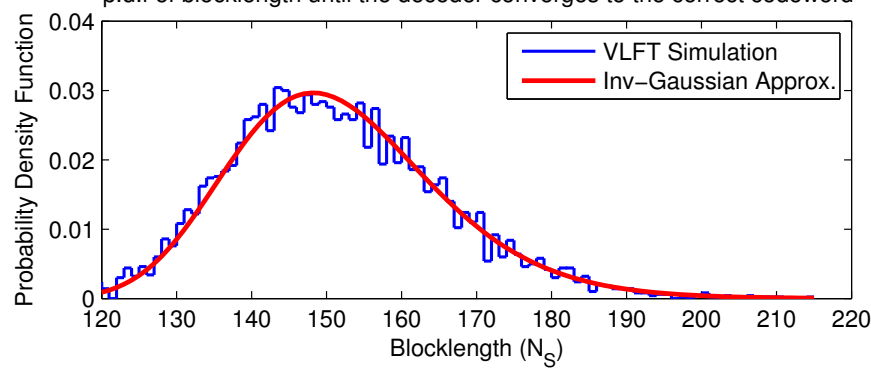

Fig. 1: Histogram of VLFT correct decoding blocklength for GF(256) NB-LDPC for SNR $2.0 \mathrm{~dB}$ and inverse-Gaussian approximation with $\mu_{S}=0.63$ and $\sigma_{S}^{2}=0.057$. Smallest blocklength is $N_{0}=120$ bits, $k=96$ bits, $R_{0}=\frac{k}{N_{0}}=0.8$.

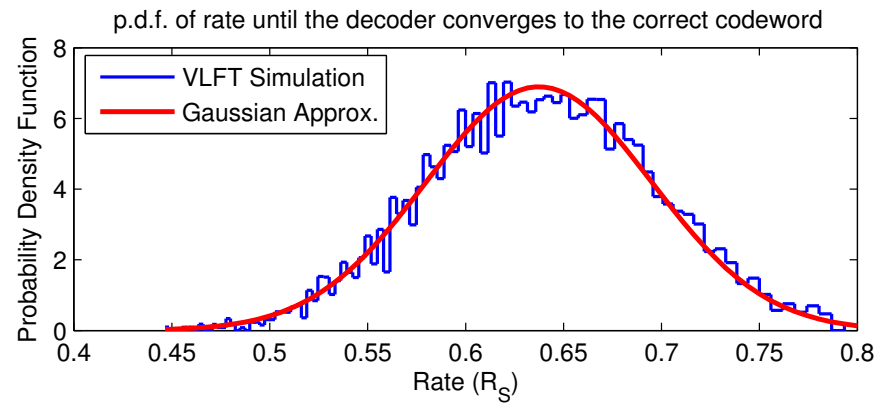

Fig. 2: Histogram of $R_{S}=\frac{k}{N_{S}}$ computed from Fig. 1 and Gaussian approximation with $\mu_{S}=0.63$ and $\sigma_{S}^{2}=0.057$.

transmitter sends one bit at a time until the decoder decodes correctly. In [6], to maximize throughput the initial code-rate of the NB-LDPC code is chosen so that almost no codeword is successfully decoded in the initial transmission. Thus, the histogram of the number of additional increments required to decode correctly does not have a spike at zero.

For the system of [6], the "VLFT simulation" plot in Fig. 1 shows the empirical probability mass function (p.m.f.) of the total blocklength $\left(N_{S}\right)$, which includes the size of the initial block and each of the incremental redundancies, required for the NB-LDPC code to decode to the transmitted codeword. The "VLFT simulation" plot in Fig. 2 shows the empirical histogram of the instantaneous rate $\left(R_{S}=\frac{k}{N_{S}}\right)$ at which decoding is successful. Fig. 2 shows that $R_{S}$ is wellapproximated by the Gaussian distribution

$$
f_{R_{S}}(r)=\frac{1}{\sqrt{2 \pi \sigma_{S}^{2}}} e^{-\frac{\left(r-\mu_{S}\right)^{2}}{2 \sigma_{S}^{2}}}
$$

with mean $\mu_{S}=E\left(R_{S}\right)$ and variance $\sigma_{S}^{2}=\operatorname{var}\left(R_{S}\right)$. This is consistent with the celebrated accuracy of the "normal approximation" of information density in [8], so is not unexpected.

The parameters $\mu_{S}=E\left(R_{S}\right)$ and $\sigma_{S}^{2}=\operatorname{var}\left(R_{S}\right)$ for a particular code need to be determined through simulation and curve fitting as above. In general $\mu_{S}$ will be less than the channel capacity because of the sub-optimality of the code. In this case capacity is 0.6422 bits and $\mu_{S}=0.63$ bits.
Note that $\mu_{S}$ is not the expected throughput but rather the average of the instantaneous rates supported by the channel. The cumulative distribution function (c.d.f.) of $N_{S}$ is $F_{N_{S}}(n)=P\left(N_{S} \leq n\right)$, and we have

$$
F_{N_{S}}(n)=P\left(\frac{k}{R_{S}} \leq n\right)=P\left(R_{S} \geq \frac{k}{n}\right)=1-F_{R_{S}}\left(\frac{k}{n}\right) .
$$

Taking the derivative of $F_{N_{S}}$ using the Gaussian approximation of $F_{R_{S}}$ produces the following "inverse-Gaussian" approximation for p.d.f. of $N_{S}$ :

$$
f_{N_{S}}(n)=\frac{k}{n^{2} \sqrt{2 \pi \sigma_{S}^{2}}} e^{\frac{-\left(\frac{k}{n}-\mu_{S}\right)^{2}}{2 \sigma_{S}^{2}}} .
$$

As shown in Fig. 1, this p.d.f closely approximates the empirical distribution of $N_{S}$. For $N_{1}<N_{2}$, the probability of the decoding attempt being successful at blocklength $N_{2}$ but not at $N_{1}$ using this approximation is

$$
\begin{aligned}
\int_{N_{1}}^{N_{2}} f_{N_{S}}(n) d n & =\int_{N_{1}}^{N_{2}} \frac{k}{n^{2} \sqrt{2 \pi \sigma_{S}^{2}}} e^{\frac{-\left(\frac{k}{n}-\mu_{S}\right)^{2}}{2 \sigma_{S}^{2}}} d n \\
& =Q\left(\frac{\frac{k}{N_{2}}-\mu_{S}}{\sigma_{S}}\right)-Q\left(\frac{\frac{k}{N_{1}}-\mu_{S}}{\sigma_{S}}\right) .
\end{aligned}
$$

The increase in blocklength from $N_{1}$ to $N_{2}$ reduces the rate from $\frac{k}{N_{1}}$ to $\frac{k}{N_{2}}$. Note that (5) gives the probability that the channel supports rate $\frac{k}{N_{2}}$ while not supporting the higher rate $\frac{k}{N_{1}}$. The $Q$ functions in (5) are due to the normally-distributed success rate $\left(R_{S}\right)$ at $\frac{k}{N_{1}}$ and $\frac{k}{N_{2}}$.

\section{VLFT WITH LIMITED NUMBER OF TRANSMISSIONS}

Using the p.d.f of $N_{S}$ from (3) we find the optimal blocklengths $\left\{N_{1}, N_{2}, \ldots, N_{m}\right\}$ to maximize the throughput when at most $m$ transmissions can be accumulated at the receiver. Initial blocklength $N_{1}$ satisfies $N_{1} \geq N_{0}$ where $N_{0}$ is the smallest possible blocklength (of the original NB-LDPC code). Each of the additional $N_{1}-N_{0}$ bits transmitted in the first transmission are the exclusive-or of all eight bits representing one of the variable nodes of the original rate- $k / N_{0}$ NB-LDPC code. For $i>1$ the $N_{i}-N_{i-1}$ incremental bits are not from a predetermined rate-compatible code. Rather they are determined using active feedback as described in detail in [6].

If decoding is not successful after the $m^{t h}$ decoding attempt, the accumulated transmissions are forgotten and transmission starts over with a new transmission of the first block of $N_{1}$ symbols. Define the throughput as $R_{T}=\frac{E(K)}{E(N)}$, where $E(K)$ is the effective number of information bits transferred correctly over the channel in one accumulation cycle (AC) and $E(N)$ represents the expected number of channel uses in one $\mathrm{AC}$.

The expression for $E(N)$ is

$$
\begin{aligned}
& E(N)=N_{1} Q\left(\frac{\frac{k}{N_{1}}-\mu_{S}}{\sigma_{S}}\right) \\
& +\sum_{i=2}^{m} N_{i}\left[Q\left(\frac{\frac{k}{N_{i}}-\mu_{S}}{\sigma_{S}}\right)-Q\left(\frac{\frac{k}{N_{i-1}}-\mu_{S}}{\sigma_{S}}\right)\right] \\
& +N_{m}\left[1-Q\left(\frac{\frac{k}{N_{m}}-\mu_{S}}{\sigma_{S}}\right)\right]
\end{aligned}
$$


The right hand side of (6) shows the expected latency for successful decoding on the first attempt in the AC. $Q\left(\frac{\frac{k}{N_{1}}-\mu_{S}}{\sigma_{S}}\right)$ is the probability decoding successfully with the initial block of $N_{1}$. Similarly, the terms in (7) account for the latency of decoding that is first successful at total blocklength $N_{i}$ (at the $i^{\text {th }}$ decoding attempt). Finally, the probability of not being able to decode even at $N_{m}$ is $1-Q\left(\frac{\frac{k}{N_{m}}-\mu_{S}}{\sigma_{S}}\right)$ which is shown in (8). Even when the decoding has not been successful at $N_{m}$, the channel has been used for $N_{m}$ channel symbols.

The expected number of successfully transferred information bits $E(K)$ is

$$
E(K)=k Q\left(\frac{\frac{k}{N_{m}}-\mu_{S}}{\sigma_{S}}\right),
$$

where $Q\left(\frac{\frac{k}{N_{m}}-\mu_{S}}{\sigma_{S}}\right)$ is the probability of successful decoding at some point in the $\mathrm{AC}$. Note that $E(K)$ depends only upon $N_{m}$. In fact, $E(K) \approx k$ and thus not sensitive to the choice of $N_{m}$ for large values of $N_{m}$.

We optimize $\left\{N_{1}, N_{2}, \ldots, N_{m}\right\}$ to maximize $R_{T}=\frac{E(K)}{E(N)}$. The order of complexity for the exhaustive search (ES) algorithm is $O\left(\left(\begin{array}{c}N_{\max }-N_{0} \\ m\end{array}\right)\right)$, where $N_{\max }$ is the maximum allowable overall blocklength for an AC. Since $E(K) \approx k$, maximization of $R_{T}$ is equivalent to minimization of $E(N)$.

We introduce sequential differential approximation (SDA) as an alternative to ES. Over the range of possible $N_{1}$ values, $\left\{N_{2}, \ldots, N_{m}\right\}$ are optimized to minimize $E(N)$ for each fixed value of $N_{1}$ by setting derivatives to zero as follows:

$$
\left\{N_{2}, \ldots, N_{m}: \frac{\partial E(N)}{\partial N_{i}}=0, \forall i=1, \ldots, m-1\right\} .
$$

For each $i \in\{2, \ldots, m\}$, the optimal value of $N_{i}$ is found by setting $\frac{\partial E(N)}{\partial N_{i-1}}=0$, yielding a sequence of relatively simple computations. In other words, we select the $N_{i}$ that makes our previous choice of $N_{i-1}$ optimal in retrospect. Thus, to find $\mathrm{N}_{2}$ we compute the derivative

$$
\frac{\partial E(N)}{\partial N_{1}}=Q\left(\frac{\frac{k}{N_{1}}-\mu_{S}}{\sigma_{S}}\right)+\left(N_{1}-N_{2}\right) Q^{\prime}\left(\frac{\frac{k}{N_{1}}-\mu_{S}}{\sigma_{S}}\right)=0
$$

and solve for $N_{2}$ where

$$
Q^{\prime}\left(\frac{\frac{k}{N_{i}}-\mu_{S}}{\sigma_{S}}\right)=\frac{k}{N_{i}^{2} \sigma_{S}} \frac{1}{\sqrt{2 \pi}} e^{\frac{\left(\frac{k}{N_{i}}-\mu_{S}\right)^{2}}{2 \sigma_{S}^{2}}} .
$$

For $i>2, \frac{\partial E(N)}{\partial N_{i-1}}=0$ depends only on $\left\{N_{i-2}, N_{i-1}, N_{i}\right\}$ as follows:

$\frac{\partial E(N)}{\partial N_{i-1}}=Q\left(\frac{\frac{k}{N_{i-1}}-\mu}{\sigma}\right)+\left(N_{i-1}-N_{i}\right) Q^{\prime}\left(\frac{\frac{k}{N_{i-1}}-\mu}{\sigma}\right)-Q\left(\frac{\frac{k}{N_{i-2}}-\mu}{\sigma}\right)$.

Thus we can solve for $N_{i}$ as

$$
\left.N_{i}=\frac{Q\left(\frac{\frac{k}{N_{i-1}}-\mu}{\sigma}\right)+N_{i-1} Q^{\prime}\left(\frac{\frac{k}{N_{i-1}}-\mu}{\sigma}\right)-Q\left(\frac{\frac{k}{N_{i-2}}-\mu}{\sigma}\right)}{Q^{\prime}\left(\frac{k}{N_{i-1}}-\mu\right.}\right) .
$$

TABLE I: Optimized $\left\{N_{1}, N_{2}, \ldots, N_{m}\right\}, R_{T}$, and $\lambda$ from ES and SDA for $k=96$ bits for VLFT on a $2 \mathrm{~dB}$ SNR binary-input AWGN channel using $\mu_{S}=0.63$ and $\sigma_{S}^{2}=0.057$.

\begin{tabular}{l|c|l|l|l} 
Alg. & $m$ & $\left\{N_{1}, N_{2}, \ldots, N_{m}\right\}$ & $R_{T}$ & $\lambda$ \\
\hline \hline ES, SDA & 2 & 158,188 & 0.566 & 169.6 \\
\hline ES & 3 & $150,167, \mathbf{1 9 4}$ & 0.58638 & 163.71 \\
SDA & 3 & $150,167, \mathbf{1 9 5}$ & 0.58635 & 163.72 \\
\hline ES & 4 & $146,158,172, \mathbf{1 9 8}$ & 0.59709 & 160.77 \\
SDA & 4 & $146,158,172, \mathbf{1 9 7}$ & 0.59707 & 160.78 \\
\hline ES, SDA & 5 & $143,153,163,176,201$ & 0.603 & 159.2 \\
\hline ES, SDA & 6 & $140,149,157,166,179,204$ & 0.608 & 157.9 \\
\hline ES, SDA & 7 & $139,147,154,161,170,182,206$ & 0.611 & 157.1
\end{tabular}

TABLE II: Optimized $R_{T}$, and $\lambda$ using SDA for various values of $m$ with $k=96$ bits for VLFT on a $2 \mathrm{~dB}$ SNR binary-input AWGN channel using $\mu_{S}=0.63$ and $\sigma_{S}^{2}=0.057$.

\begin{tabular}{c|c|c|c|c|c|c|c}
$m$ & 2 & 3 & 5 & 10 & 15 & 20 & $\infty$ \\
\hline$\lambda$ & 167.1 & 163.7 & 159 & 155.5 & 154.3 & 153.7 & 151.9 \\
\hline$R_{T}$ & 0.566 & 0.586 & 0.603 & 0.618 & 0.622 & 0.624 & 0.632
\end{tabular}

Table I shows optimized $\left\{N_{1}, N_{2}, \ldots, N_{m}\right\}$ and resulting throughput $R_{T}$ and expected latency $\lambda=k / R_{T}$ for various $m$. The values obtained by SDA are very close to the values obtained by ES.

For $m=2,5,6$, and 7 , the optimized blocklengths for both approaches are the same. For $m=3$ and 4 the blocklengths differ only in the value of $N_{m}$ (shown in bold) and only by one bit. This small difference in $N_{m}$ causes a negligible difference in the maximum throughput $R_{T}$ and minimum latency $\lambda=\frac{k}{R_{T}}$.

Table II shows the optimum $R_{T}$ and $\lambda$ for various $m$ using SDA. Since the complexity of ES is exponential in $m$, it is infeasible to obtain a globally optimal solution for $m>7$. whereas SDA, with complexity $O\left(N_{\max }-N_{0}\right)$, can find a solution within seconds even for large $m$.

\section{TWO-PHASE VLF}

Now we consider the two-phase VLF model in which the transmitter uses the primary communication channel to confirm whether the receiver has decoded to the correct codeword. As in [5] the two-phase incremental redundancy scheme has each communication phase followed by a confirmation phase.

Fig. 3 shows a block diagram for the two-phase communication scheme. Starting at the left, a message block of size $N_{1}$ is transmitted. If the decoder decodes correctly, the transmitter sends a coded forward "ACK" on the same noisy channel to confirm the successful decoding. If the receiver decodes incorrectly, the transmitter sends a coded forward NACK. The ACKs and NACKs are repetition codes of length $A_{1}$ symbols.

Since the receiver and transmitter run the same decoder on the same received channel symbols, if the decoder does not converge to any codeword with $N_{1}$ symbols, the transmitter skips the unnecessary confirmation phase and immediately transmits the second increment $\left(N_{2}-N_{1}\right)$. The transmitter terminates transmission when either the receiver decodes both the message and the following ACK correctly or when the 


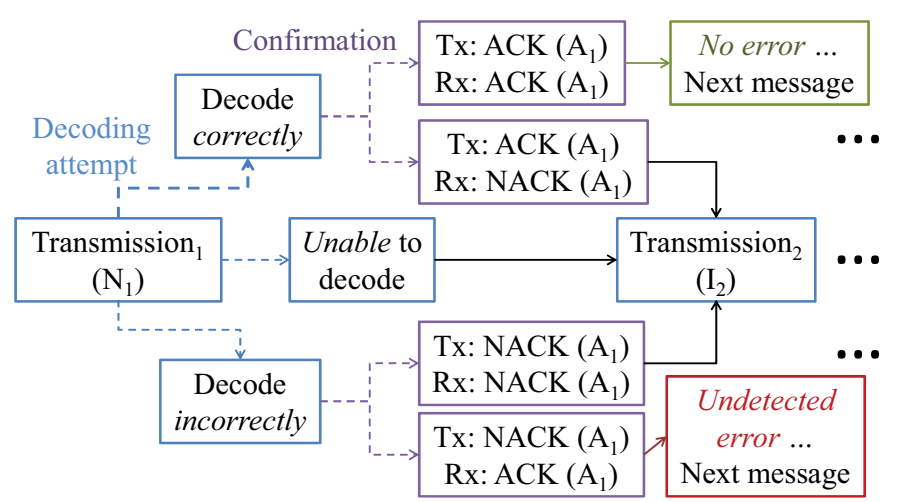

Fig. 3: Two-phase VLF block diagram

receiver decodes both the message the subsequent NACK incorrectly. The latter scenario is the only case that results in an error, and this error is undetected by the receiver. The blocklengths of each transmission and confirmation message are chosen to guarantee a probability of undetected error of at most $\epsilon$. If the message is not decoded correctly even after $m$ transmissions (and the NACKs are correctly received), the receiver deletes all received symbols and a new AC begins with the transmitter sending the original block of $N_{1}$ symbols.

Since the transmitter sends NACK only when a wrong codeword is decoded, it is crucial to differentiate between erroneous decoding and failure to converge to a codeword. Fig. 4 shows the histogram of the required cumulative number of symbols until the receiver stops converging to an incorrect codeword $\left(N_{E}\right)$. Note that Fig. 4 is conditioned on the decoder initially decoding to a wrong codeword at $N_{0}=120$. The probability that the decoder decodes incorrectly at $N_{0}$ is $\alpha$, and $\alpha=0.165$ for the experiment that produced the histogram in Fig. 4 . For blocklengths larger than $N_{E}$, the decoder either decodes correctly or fails to converge to any codeword. This is a different condition than correct decoding, the distribution is well-modeled by an inverse Gaussian as in Fig. 1. Fig. 5 shows the histogram of $R_{E}=\frac{k}{N_{E}}$, the instantaneous rate at which the decoder stops decoding to the wrong codeword, and the corresponding Gaussian approximation.

In the two-phase VLF setting, we use the probability distributions of $N_{S}, R_{S}, N_{E}$ and $R_{E}$ from Figs. 1, 2, 4, and 5. The optimization problem is to maximize $R_{T}=\frac{E(K)}{E(N)}$ where

$$
E(K)=k\left(Q\left(\frac{\frac{k}{N_{m}}-\mu_{S}}{\sigma_{S}}\right)-\sum_{i=1}^{m} P_{i}^{E E}\right),
$$

but as in Sec. II we assume $E(K) \approx k$, and

$$
\begin{aligned}
E(N)= & \sum_{i=1}^{m}\left(N_{i}+A_{i}\right)\left[P_{i}^{S S}+P_{i}^{E E}\right]+A_{i}\left[P_{i}^{S E}+P_{i}^{E S}\right] \\
& +N_{m}\left(1-Q\left(\frac{\frac{k}{N_{m}}-\mu_{S}}{\sigma_{S}}\right)+P_{m}^{S E}\right) .
\end{aligned}
$$

We maximize $R_{T}$ under the constraint that $\sum_{i=1}^{m} P_{i}^{E E}<\epsilon$, where $P_{i}^{E E}$ represents the probability the receiver decodes

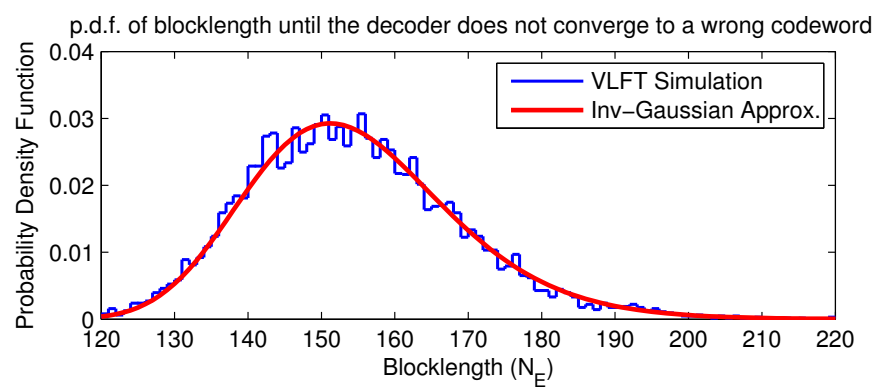

Fig. 4: Empirical histogram and inverse-Gaussian fit for the cumulative blocklength $\left(N_{E}\right)$ to stop decoding incorrectly.

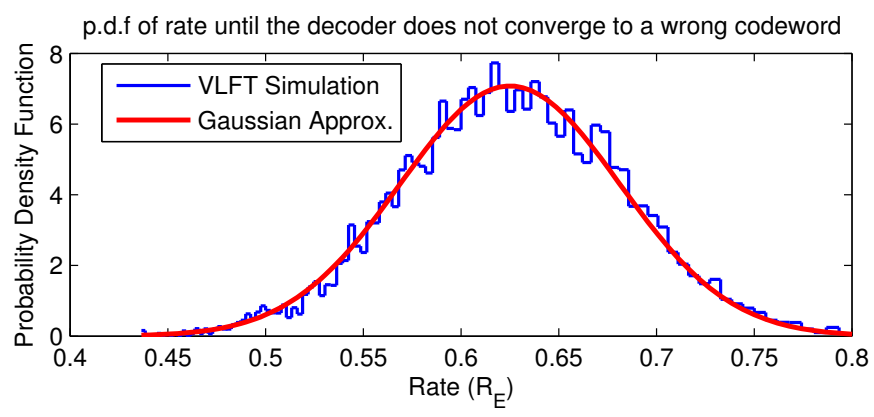

Fig. 5: Empirical histogram and Gaussian approximation with $\mu_{E}=0.626$ and $\sigma_{E}^{2}=0.056$ of $R_{E}$ in VLFT setting.

both the message and the NACK erroneously. $P_{i}^{S S}$ is the probability the receiver decodes both message and ACK successfully. The term multiplying $N_{m}$ in (16) is the probability that an AC ends without satisfying either of these stopping conditions. $P_{i}^{S E}$ is the probability of decoding the message successfully but decoding the ACK as a NACK. Conversely, $P_{i}^{E S}$ is the probability of decoding the message erroneously but decoding the NACK successfully. These probabilities are computed as follows:

$$
\begin{aligned}
& P_{i}^{S S}=\left[Q\left(\frac{\frac{k}{N_{i}}-\mu_{S}}{\sigma_{S}}\right)-Q\left(\frac{\frac{k}{N_{i-1}}-\mu_{S}}{\sigma_{S}}\right)\right]\left[1-Q\left(\frac{\sqrt{A_{i}}}{\sigma_{c}}\right)\right] \\
& P_{i}^{E E}=\left[\alpha\left(1-Q\left(\frac{\frac{k}{N_{i}}-\mu_{E}}{\sigma_{E}}\right)\right)\right]\left[Q\left(\frac{\sqrt{A_{i}}}{\sigma_{c}}\right)\right] \\
& P_{i}^{S E}=\left[Q\left(\frac{\frac{k}{N_{i}}-\mu_{S}}{\sigma_{S}}\right)-Q\left(\frac{\frac{k}{N_{i-1}}-\mu_{S}}{\sigma_{S}}\right)\right]\left[Q\left(\frac{\sqrt{A_{i}}}{\sigma_{c}}\right)\right] \\
& P^{E S}=\left[\alpha\left(1-Q\left(\frac{\frac{k}{N_{i}}-\mu_{E}}{\sigma_{E}}\right)\right)\right]\left[\left(1-Q\left(\frac{\sqrt{A_{i}}}{\sigma_{c}}\right)\right)\right] \text {. }
\end{aligned}
$$

In (17) the probability of decoding correctly at $N_{i}$ and not at $N_{i-1}$ is $Q\left(\frac{\frac{k}{N_{i}}-\mu_{S}}{\sigma_{S}}\right)-Q\left(\frac{\frac{k}{N_{i}-1}-\mu_{S}}{\sigma_{S}}\right)$ and $Q\left(\frac{\sqrt{A_{1}}}{\sigma_{c}}\right)$ is the probability that the ACK is decoded as a NACK, where $\sigma_{c}$ is the standard deviation of the channel noise. In (18), $\alpha\left[1-Q\left(\frac{\frac{k}{N_{i}}-\mu_{E}}{\sigma_{E}}\right)\right]$ is the probability of decoding erroneously at $N_{i}$. 
TABLE III: Optimized $\left\{N_{1}, \ldots, N_{m}\right\}$ for $m=5$ two-phase VLF using SDA and ES with $\left\{A_{1}, \ldots, A_{5}\right\}=\{5,4,3,3,3\}$.

\begin{tabular}{c|c|c|c} 
Alg. & $k$ & $\left\{N_{1}, N_{2}, \ldots, N_{5}\right\}$ & $\epsilon$ \\
\hline SDA & 96 & $145,156,167,180,202$ & $1.2 \mathrm{E}-3$ \\
SDA & 96 & $146,158,171,188,230$ & $9.4 \mathrm{E}-4$ \\
\hline ES & 96 & $146,158,170,184,211$ & $9.9 \mathrm{E}-4$
\end{tabular}

To optimize the blocklengths for two-phase VLF we used both ES and SDA approaches from Sec. III for fixed values of $\left\{A_{1}, \ldots, A_{m}\right\}$. Table III shows two sets of $\left\{N_{1}, \ldots, N_{m}\right\}$ obtained for different $N_{1}$ in SDA with $\epsilon \approx 10^{-3}$. The optimized $\left\{N_{1}, \ldots, N_{m}\right\}$ with $\epsilon \leq 10^{-3}$ from ES is close to the SDA optimized blocklengths. The optimized blocklengths from SDA can also be used as optimization limits for ES algorithm and significantly reduce the ES optimization space.

\section{RESULTS}

Table IV summarizes the blocklengths that maximize the throughput in the two-phase VLF setting with $\epsilon=10^{-3}$, for both NB-LDPC codes and tail-biting convolutional codes. Blocklengths for the NB-LDPC codes are obtained from Eqns. (14-16) and blocklengths for the convolutional codes are based on the coordinate-descent algorithm in [7] using the assumption of rate-compatible sphere-packing.Table IV also shows the percentage of BIAWGN capacity obtained in the two-phase VLF setting with $m=5$ transmissions. The rate- $1 / 3$ convolutional codes (CC) have octal generator polynomials $(117,127,155)$ for the 64-state code and $(2325,2731,3747)$ for the 1024-state code. The NB-LDPC codes are described completely at http://www.seas.ucla.edu/csl/resources/index.htm.

Fig. 6 shows $R_{T}$ versus $\lambda$ for NB-LDPC and convolutional codes (CC) in VLFT and two-phase VLF settings. In VLFT with unlimited number of transmissions (1-bit increments), CCs with ML decoders perform very well at short latencies of up to 200 bits. VLFT schemes have throughputs greater than the capacity at short blocklengths because of the NTC. VLFT with NB-LDPC codes outperforms CCs at larger latencies because the codeword error rate of CCs increases once the blocklength exceeds twice the traceback depth.

Fig. 6 also shows the throughput obtained in the two-phase VLF setting for NB-LDPC codes, 64-state and 1024-state tailbiting convolutional codes with $m=5$ and $\epsilon=10^{-3}$. As the blocklength increases, as mentioned in [9], the performance of the codes in VLF gets closer to the performance in VLFT.

\section{CONCLUSION}

This paper uses the inverse-Gaussian approximation for the blocklenth of first successful decoding to optimize the size of each incremental transmission for non-binary (NB) LDPC codes to maximize throughput in VLFT and two-phase VLF settings. In the 300-500 symbol average blocklength regime, this paper reports the best VLFT and VLF throughputs yet. VLFT throughputs are higher than VLF, but VLF is more practical because it does not require NTC. For two-phase VLF with $m=5$, NB-LDPC codes with optimized blocklengths
TABLE IV: Optimized $\left\{N_{1}, \ldots, N_{m}\right\}$ for two-phase VLF with $m=5$ at SNR $2 \mathrm{~dB}$, and simulation results for $R_{T}$ and $\lambda$. $\left\{A_{1}, \ldots, A_{5}\right\}=\{5,4,3,3,3\}$ for NB-LDPC. For the CCs, $A_{i}=6,8$, and $9 \forall i$ for $k=96,192$, and 288 bits, respectively.

\begin{tabular}{c|c|c|c|c|c} 
Code & $k$ & $\left\{N_{1}, N_{2}, \ldots, N_{5}\right\}$ & $\lambda$ & $R_{T}$ & $\%$ \\
\hline NB-LDPC & 96 & $146,158,170,184,211$ & 170.4 & 0.563 & 87.7 \\
1024-CC & 96 & $138,153,166,180,204$ & 168.6 & 0.569 & 88.6 \\
\hline NB-LDPC & 192 & $301,322,344,369,408$ & 330.5 & 0.581 & 90.5 \\
1024-CC & 192 & $287,309,331,352,384$ & 349.4 & 0.549 & 85.4 \\
\hline NB-LDPC & 288 & $459,487,518,550,597$ & 495.7 & 0.581 & 90.5 \\
1024-CC & 288 & $416,441,463,488,532$ & 599.6 & 0.480 & 74.8
\end{tabular}

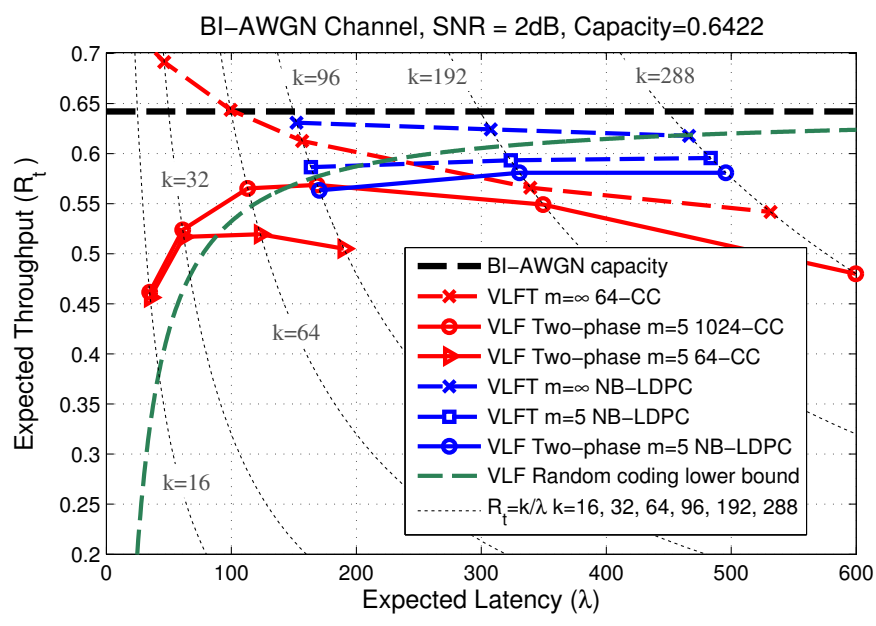

Fig. 6: $R_{T}$ vs. $\lambda$ for NB-LDPC, 64/1024-state CCs for VLFT with $m=\infty$ and $m=5$, and two-phase VLF with $m=5$.

achieve greater than $90 \%$ of the capacity of the $2 \mathrm{~dB}$ BI-AWGN channel in the 300-500 symbol range of average blocklength.

\section{REFERENCES}

[1] C. E. Shannon, "The zero error capacity of a noisy channel," IRE Trans. Inf. Theory, vol. 2, no. 3, pp. 8-19, Sep. 1956.

[2] Y. Polyanskiy, H. V. Poor, and S. Verdú, "Feedback in the non-asymptotic regime," IEEE Trans. Inf. Theory, vol. 57, no. 8, pp. 4903-4925, Aug. 2011.

[3] T.-Y. Chen, N. Seshadri, and R. Wesel, "A sphere-packing analysis of incremental redundancy with feedback," in 2011 IEEE Int. Conf. Commun. (ICC), June 2011, pp. 1-5.

[4] A. R. Williamson, M. J. Marshall, and R. D. Wesel, "Reliability-output decoding of tail-biting convolutional codes," IEEE Trans. Commun., to be published. Available: http://arxiv.org/abs/1312.1024.

[5] A. Williamson, T.-Y. Chen, and R. Wesel, "Firing the genie: Twophase short-blocklength convolutional coding with feedback," in 2013 Inf. Theory and Applications Workshop (ITA), 2013, pp. 1-6.

[6] K. Vakilinia, T.-Y. Chen, S. V. S. Ranganathan, A. R. Williamson, D. Divsalar, and R. D. Wesel, "Short-blocklength non-binary LDPC codes with feedback-dependent incremental transmissions," in Proc. 2014 IEEE Int. Symp. Inf. Theory (ISIT), Honolulu, HI, USA, July 2014.

[7] A. R. Williamson, "Reliability-output decoding and low-latency variablelength coding schemes for communication with feedback," Ph.D. dissertation, Dept. Elec. Eng., UCLA, Los Angeles, CA, 2014.

[8] Y. Polyanskiy, H. V. Poor, and S. Verdú, "Channel coding rate in the finite blocklength regime," IEEE Trans. Inf. Theory, vol. 56, no. 5, pp. 2307-2359, May 2010.

[9] T.-Y. Chen, A. R. Williamson, N. Seshadri, and R. D. Wesel, "Feedback communication systems with limitations on incremental redundancy," submitted for publication. Available: http://arxiv.org/abs/1309.0707. 